\title{
A Folding-Based Electrochemical Aptasensor for the Single-Step Detection of the SARS-CoV-2 Spike Protein
}

Federica Curti, ${ }^{a, b}$ Simone Fortunati, ${ }^{a}$ Wolfgang Knoll, ${ }^{b, c}$ Marco Giannetto, ${ }^{a}$ Roberto Corradini, ${ }^{a}$ Alessandro Bertucci, ${ }^{a *}$ and Maria Careri ${ }^{a}$

a Department of Chemistry, Life Sciences and Environmental Sustainability, University of Parma, 43124 Parma, Italy.

b Biosensor Technologies, AIT-Austrian Institute of Technology GmbH, Konrad-LorenzStraße 24, 3430 Tulln an der Donau, Austria

${ }^{c}$ Department of Scientific Coordination and Management, Danube Private University, A3500 Krems, Austria

* Corresponding Author Email:

alessandro.bertucci@unipr.it

ABSTRACT Efficient and timely testing has taken center stage in the management, control and monitoring of the current COVID-19 pandemic. Simple, rapid, cost-effective diagnostics are needed that can complement current polymerase chain reaction (PCR)based methods and lateral flow immunoassays. Here, we report the development of an electrochemical sensing platform based on single-walled carbon nanotube screen-printed electrodes (SWCNT-SPEs) functionalized with a redox-tagged DNA aptamer that specifically binds to the receptor binding domain (RBD) of the SARS-CoV-2 spike protein S1 subunit. Single-step, reagentless detection of the S1 protein is achieved through a 
binding-induced, concentration-dependent folding of the DNA aptamer that reduces the efficiency of the electron transfer process at the electrode surface and causes a suppression of the resulting amperometric signal. This aptasensor is specific for the target S1 protein with a dissociation constant $\left(K_{D}\right)$ value of $43 \pm 4 \mathrm{nM}$ and a limit of detection (LOD) of $7 \mathrm{nM}$. We demonstrate that the target S1 protein can be detected both in a buffer solution and in an artificial viral transport medium widely used for the collection of nasopharyngeal swabs, and that no cross-reactivity is observed in the presence of different, non-target viral proteins. This SWCNT-SPE-based format of electrochemical aptasensor may be extended to the detection of other protein targets for which nucleic acid aptamer ligands are made available.

\section{INTRODUCTION}

The COVID-19 pandemic caused by the spread of the severe acute respiratory syndrome coronavirus 2 (SARS-CoV-2) continues to claim victims and to determine disruptions in healthcare systems, economies, and social life worldwide. ${ }^{1-3}$ The deployment at a fast pace of different COVID-19 vaccines has had a huge impact on the pandemic by offering protection from severe and acute forms of the disease and therefore helping reduce hospitalization and mortality. ${ }^{4,5}$ Nevertheless, vaccines alone are not able to contain the spread of the virus, and complementary measures must be enforced. ${ }^{6,7}$ Efficient and focused testing is necessary for timely spotting SARS-CoV-2 infection, monitoring the diffusion of the disease, and curbing transmission of the virus. ${ }^{8,9}$ COVID diagnostics has taken center stage in everyday life, especially where proof of a negative test is a requirement for traveling and for accessing public and private spaces. ${ }^{10,11}$ Currently, 
polymerase chain reaction (PCR) is the gold standard method to detect SARS-CoV-2 infection, enabling quantification of viral RNA with high sensitivity and specificity. However, PCR is reagent-intensive and requires trained personnel and relatively expensive instrumentation. This leads, on the one hand, to waiting times that are not compatible with the highly frequent testing enforced in wealthy countries, and determines, on the other hand, a series of practical obstacles to a widespread application in lowresource settings. ${ }^{12,13}$ Cost-effective, time-saving point-of-care (POC) tests that can complement PCR-based methods are therefore much needed. Lateral flow immunoassays enable the rapid detection of a SARS-CoV-2 antigen by using low-cost, portable hardware.$^{14}$ However, their relatively low sensitivity and specificity is a limit to their ability to deliver unambiguous and accurate diagnostics, and therefore to their potential to guide healthcare and policy measures. ${ }^{15}$ Alternatively, electrochemical biosensors are easy-to-use and cost-effective platforms that can be engineered into POC diagnostic devices for the rapid quantification of specific targets. ${ }^{16-18}$ Several electrochemical COVID-19 immunosensors were recently developed that enabled ultrasensitive detection of SARS-CoV-2 antigen proteins. ${ }^{19-21}$ A particular format of electrochemical sensors is E-DNAs. These are rapid, simple, reagent-free sensors that leverage target-induced conformational or structural changes in a DNA-based architecture or in a DNA aptamer to generate a measurable output following variation of the electron transfer efficiency between a redox reporter and the electrode surface..$^{22-24}$ In the context of COVID-19, Shana O. Kelley and coworkers achieved detection of viral particles within 5 minutes through a chronoamperometry strategy based on electrodes modified with hybrid DNA-antibody receptors targeting the SARS-CoV-2 spike (S) protein 
displayed on the virion surface. ${ }^{25}$ In aptamer-based E-DNAs, an electrochemical signal is generated when a binding-induced change in the structure folding of a redox-tagged aptamer leads to a change in the relative position of the redox reporter with respect to the electrode surface ${ }^{26}$ Recently, Idili et al. applied this strategy to COVID-19 diagnosis and performed electrochemical detection of the SARS-CoV-2 $S$ protein by using gold electrodes modified with a DNA aptamer engineered to undergo a binding-induced conformational change. ${ }^{27}$ In such a context, here we report the development on a electrochemical sensing platform based on cheap, commercial single-walled carbon nanotube screen-printed electrodes (SWCNT-SPEs) functionalized with a redox-tagged DNA aptamer selected against the receptor binding domain (RBD) of the SARS-CoV-2 spike protein S1 subunit. ${ }^{28}$ Binding-induced folding of this DNA aptamer in the presence of the target S1 protein leads to a concentration-dependent suppression in the registered amperometric signal. We demonstrate that this aptasensor specifically recognizes and detects the target $\mathrm{S} 1$ protein both in a buffer solution and in an artificial complex matrix, requiring only a few hours of incubation and no additional reagents.

\section{EXPERIMENTAL SECTION}

2.1. Materials. Sodium chloride $(\mathrm{NaCl})$, sodium bicarbonate $\left(\mathrm{NaHCO}_{3}\right)$, disodium hydrogen phosphate $\left(\mathrm{Na}_{2} \mathrm{HPO}_{4}\right)$, potassium dihydrogen phosphate $\left(\mathrm{KH}_{2} \mathrm{PO}_{4}\right)$, sodium dodecyl sulfate (SDS), Trizma® Base, N-(3-dimethylaminopropyl)- $N$ 'ethylcarbodiimide hydrochloride (EDC), N-hydroxysuccinimide (NHS), 4morpholineethanesulfonic acid monohydrate (MES), pyrene, ethanolamine, hydrochloric acid $(\mathrm{HCl}, 37 \%)$, calcium chloride $(\mathrm{KCl})$, ethanolamine, sodium hydroxide $(\mathrm{NaOH})$, dimethyl sulfoxide (DMSO), were purchased from Sigma-Aldrich (Milan, Italy). Viral 
Transport Medium (VTM) was purchased from CleaniSciences (Guidonia Montecelio, Italy). The following synthetic probes were purchased from Metabion International AG (Plannegg, Germany): redox-tagged SARS-CoV-2 aptamer : 5' - AttoMB2 - CGC AGC ACC CAA GAA CAA GGA CTG CTT AGG ATT GCG ATA GGT TCG GTT TTT - C7 Amino - 3'; SARS-CoV-2 aptamer : 5' - CGC AGC ACC CAA GAA CAA GGA CTG CTT AGG ATT GCG ATA GGT TCG GTT TTT - C7 Amino - 3'; redox-tagged thrombin aptamer: 5' - AttoMB2 - TAA GTT CAT CTC CCC GGT TGG TGT GGT TGG T - C7 Amino - 3'; redox-tagged PDGF aptamer: 5' - AttoMB2 - CAG GCT ACG GCA CGT AGA GCA TCA CCA TGA TCC TG - C7 Amino - 3'. SARS-CoV-2 Spike protein (S1) and Influenza A H1N1 (A/California/04/2009 (H1N1)) were purchased from Twin Helix Srl (Milano, Italy). Recombinant Coronavirus Spike Protein MERS-CoV S1 was purchased from Vinci-Biochem Srl (Vinci, Italy). Buffers were prepared as follows : MES buffer: 0.1 M MES (pH adjusted to 5 with $\mathrm{NaOH}$ ); Tris Buffered Saline (TBS): $0.1 \mathrm{M}$ Trizma® Base ( $\mathrm{pH}$ adjusted to 7.4 with $\mathrm{HCl}$ ); Carbonate buffer (CB): $0.1 \mathrm{M} \mathrm{NaHCO}, 0.1 \% \mathrm{w} / \mathrm{v}$ SDS (pH adjusted to 9 with $\mathrm{NaOH}$ ); Phosphate Buffer Saline (PBS): $1.37 \mathrm{M} \mathrm{NaCl}, 0.08 \mathrm{M} \mathrm{Na}_{2} \mathrm{HPO}_{4}$, $0.027 \mathrm{M} \mathrm{KCl}, 0.012 \mathrm{M} \mathrm{KH}_{2} \mathrm{PO}_{4}$ (pH adjusted to 7.4 with $\mathrm{HCl}$ ); Reading buffer (RB): PBS. Single Walled Carbon Nanotubes Screen-Printed electrodes (SWCNT-SPEs) were purchased from Metrohm Italiana Srl (Varese, Italy).

2.2. Aptamer immobilization on SWCNT-SPEs. The SWCNT surface was initially treated with $0,2 \mathrm{M} \mathrm{EDC}$ and $0,05 \mathrm{M} \mathrm{NHS}$ in MES buffer $(50 \mu \mathrm{L})$ for 30 min to activate the carboxylic groups of the carbon nanotubes, followed by rinsing with water. Subsequently, $50 \mu \mathrm{L}$ of redox-tagged SARS-CoV-2 aptamer (500 nM) solution in CB was deposited onto the SWCNT electrode and left incubating for $2 \mathrm{~h}$. The electrode was then 
thoroughly washed with water. A capping step of 30 min using ethanolamine in TBS (50 $\mathrm{mM}$ ) was carried out to quench the unreacted active ester groups. Next, the surface was washed with TBS. A solution of pyrene as a backfilling agent in DMSO (500 nM) was deposited on the electrode $(50 \mu \mathrm{L})$ for $30 \mathrm{~min}$, after which the electrode was rinsed first with DMSO and then with water.

\subsection{Determination of the aptamer surface density. Fluorescence} spectroscopy was selected as the technique to estimate the surface density of the covalently immobilised SARS-CoV-2 aptamer. The emission of AttoMB2 was collected in solution at $\lambda_{E m}=680 \mathrm{~nm}\left(\lambda_{E x}=650 \mathrm{~nm}\right)$ before $\left(I_{i}\right)$ and after $\left(I_{f}\right)$ the aptamer immobilisation on the electrode (c.f. section 2.2). The $\Delta l_{i-f}$ percentage was used to estimate the number of aptamer probes per $\mathrm{mm}^{2}$ tethered to the CNTs. The measurements were replicated three times and the value obtained is reported as the mean value \pm standard deviation.

2.4. Detection of S1 protein. A solution of S1 protein at different concentrations $(0.3,1,3,10,25,30,50,100,300$ and $500 \mathrm{nM})$ in PBS was incubated for $2 \mathrm{~h}$, at room temperature, on the electrode surface, followed by rinsing with PBS. Next, $50 \mu \mathrm{L}$ of PBS were deposited onto the surface and the electrochemical measurement was performed by acquiring a DPV scan with the following parameters: potential range from -1.1 to -0.2 $\mathrm{V}$; step potential $+0.00495 \mathrm{~V}$; modulation amplitude $+0.04995 \mathrm{~V}$; modulation time 0.102 $\mathrm{s}$; interval time $0.4 \mathrm{~s}$. The same protocol was applied during specificity studies, when a thrombin aptamer and a PDGF aptamer, respectively, were immobilized onto the electrode surface and exposed to $\mathrm{S} 1$ protein, as well as when MERS-Cov S1 and Influenza-A H1N1 proteins were incubated onto the electrode surface previously functionalized with the SARS-CoV-2 aptamer. This protocol and acquisition parameters 
were also used for measurements of S1 protein diluted in VTM as the binding buffer (50 and $100 \mathrm{nM})$.

2.5. Competitive experiments of $\mathbf{S} 1$ protein binding. The electrode surface was functionalized as described above with a redox-tagged SARS-CoV-2 aptamer. The target S1 protein (100 nM concentration, PBS) was incubated with an unlabeled version of the same SARS-CoV-2 aptamer sequence for $2 \mathrm{~h}$ at room temperature. This solution was then transferred onto the electrode surface and left incubating for $2 \mathrm{~h}$. Then, the electrode surface was washed with PBS and $50 \mu \mathrm{L}$ of PBS were deposited onto the surface to carry out the electrochemical measurement by acquiring a DPV scan with the same parameters reported in section 2.3.

2.6. Data analysis. The current signals obtained from the electrochemical measurements as a function of the corresponding $\mathrm{S} 1$ protein concentrations were analyzed in OriginPro (OriginLab ${ }^{\mathrm{TM}}$ ) by using the Langmuir-type equation reported below:

$$
I=a+c *\left[[S 1 \text { protein }] /\left(K_{D}+[S 1 \text { protein }]\right)\right]
$$

where $K_{D}$ is the dissociation constant, $a$ and $c$ are fitting parameters of the Langmuir equation. All the measurements were replicated three times and all the figures show the mean values \pm standard deviations. The limit of detection (LOD) and limit of quantification (LOQ) were determined according to Eurachem guidelines. 


\section{RESULTS AND DISCUSSION}

3.1. Immobilization of SARS-CoV-2 DNA aptamer. Several aptamers have been recently artificially evolved that can specifically bind to the S1 protein of SARS-CoV-2 and can potentially support new therapeutic strategies for COVID-19. ${ }^{28-31}$ DNA aptamers can be used in lieu of antibodies as specific recognition elements in the development of SARS-CoV-2 biosensors, enabling a range of sensing platforms with optical or electrochemical readout. $27,29,32-35$ Besides their use as "static" synthetic receptors, aptamers are useful as "dynamic" probes in folding-based E-DNAs. In this work, we propose a novel E-DNA format for the single-step, reagent-free detection of the S1 protein that leverages cheap, commercial SWCNT-SPEs as the sensing substrate. SWNCTSPEs are a promising electrochemical platform because they are cheaper than standard gold electrodes, they show intrinsic electrocatalytic properties and a high conductivity that both improve electron transfer processes at the interface and provide enhanced amplification of current signal, and they offer a larger surface area for immobilization of an increased number of probes and receptors. ${ }^{36-38}$ The sensing mechanism of our sensor is based on a rearrangement in the redox-tagged aptamer structure induced by binding of the target S1 protein, which translates in a measurable electrochemical output. A fast electron transfer is observed in the absence of the target protein because of the $\pi-\pi$ interactions between the redox-tagged DNA aptamer and the electrode surface. In the presence of the target, the aptamer undergoes a binding-induced structural folding that reduces the electron transfer efficiency, causing a decrease in the registered amperometric current (Fig. 1). 

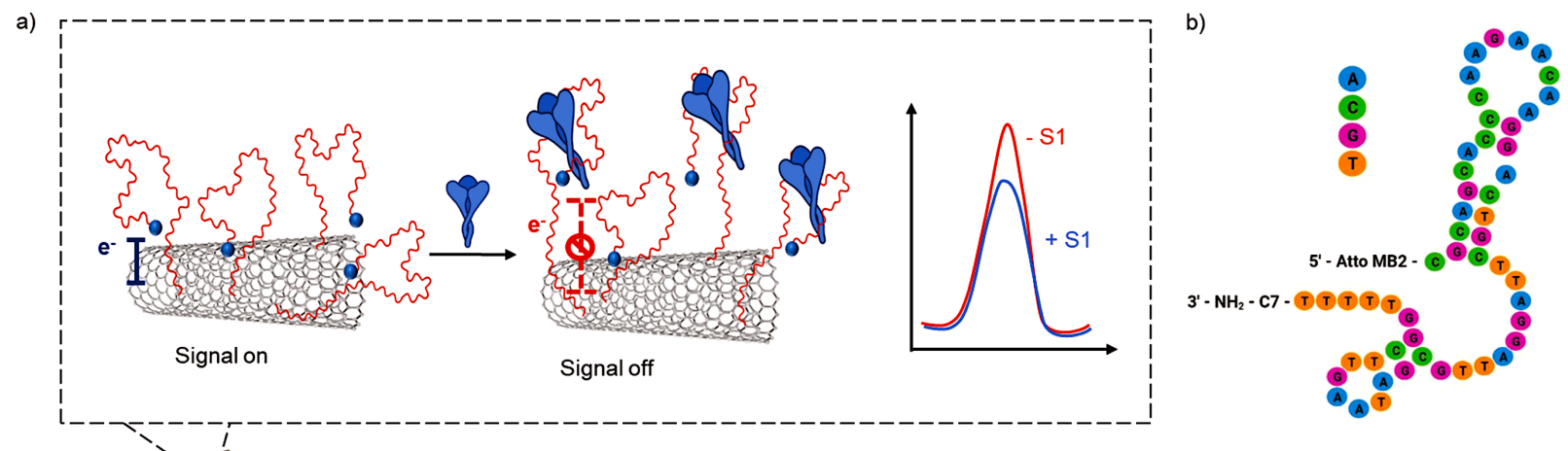

Figure 1. a) Schematic illustration of the working principle of the aptasensor based on the conformational change in a redox-tagged SARS-CoV-2 aptamer upon interaction with the target $\mathrm{S} 1$ protein. A suppression of the output current is observed in the presence of $\mathrm{S} 1$ protein because of the structural rearrangement in the aptamer folding that moves the redox reporter away from the electrode surface. b) Secondary structure of the Atto-MB2-modified SARS-CoV-2 aptamer used in this work, based on a recently discovered aptamer sequence. ${ }^{28}$

To fabricate our aptasensor, we used a modified version of a recently published SARSCoV-2 DNA aptamer that specifically binds to receptor binding domain (RBD) of the SARS-CoV-2 spike protein S1 subunit. ${ }^{28}$ We conjugated the aptamer with a redox tag (AttoMB2) at the $3^{\prime}$ terminus and introduced a free amine group at the $5^{\prime}$ terminus. This latter was used to covalently anchor the aptamers $(500 \mathrm{nM})$ to the electrode surface through the formation of amide bonds with the carboxylic groups present on the SWCNTs, which can be achieved through coupling with EDC/NHS. The coupling process was optimized by diluting the aptamer in carbonate buffer $(\mathrm{pH}=9)$ with $0.1 \%$ SDS to increase the wettability of the CNT hydrophobic surface. The surface density of the aptamer was estimated by means of fluorescence spectroscopy by following the emission of AttoMB2 
at $\lambda=680 \mathrm{~nm}$ before and after the immobilization of the aptamer onto the surface. A value of $(1.7 \pm 0.4) \cdot 10^{13}$ aptamer molecules per $\mathrm{mm}^{2}$ was obtained, which is higher than the average values generally found in the literature for E-DNAs based on gold electrode substrates. ${ }^{39,40}$ The electrode surface was eventually treated with a solution of pyrene in DMSO as a backfilling agent to minimize non-specific adsorption of the biomolecules contained in the analyzed samples. ${ }^{38}$

3.2. S1 protein-aptamer interaction analysis. The sensor was then exposed to increasing concentrations of S1 protein from 0.3 to $500 \mathrm{nM}$ in PBS buffer, and DPV voltammograms were acquired. We observed a signal-off behavior, i.e., a decrease in the amperometric signal given by the AttoMB2 redox reporter, after interaction of the aptamer with the different concentrations of the $\mathrm{S} 1$ protein, which likely depends on that AttoMB2 is moved away from the electrode surface upon target-induced folding of the DNA aptamer (Fig. 2a). The decrease in the registered amperometric current can be expressed as signal suppression \% with respect to the current signal measured in the absence of S1 protein. The obtained data were analyzed by means of a Langmuir-type binding curve model and the dissociation constant value between the aptamer attached to the electrode surface and its target S1 protein was $K_{D}=43 \pm 4 \mathrm{nM}$ (Fig. 2b). This is in good agreement with the affinity measured in solution by means of fluorescence spectroscopy reported for the original aptamer $\left(K_{D}=45 \pm 10 \mathrm{nM}\right) \cdot{ }^{28}$ It was possible to obtain a limit of detection (LOD) of $7 \mathrm{nM}$ and a limit of quantification (LOQ) of $21 \mathrm{nM}$ (Fig. 2c). 

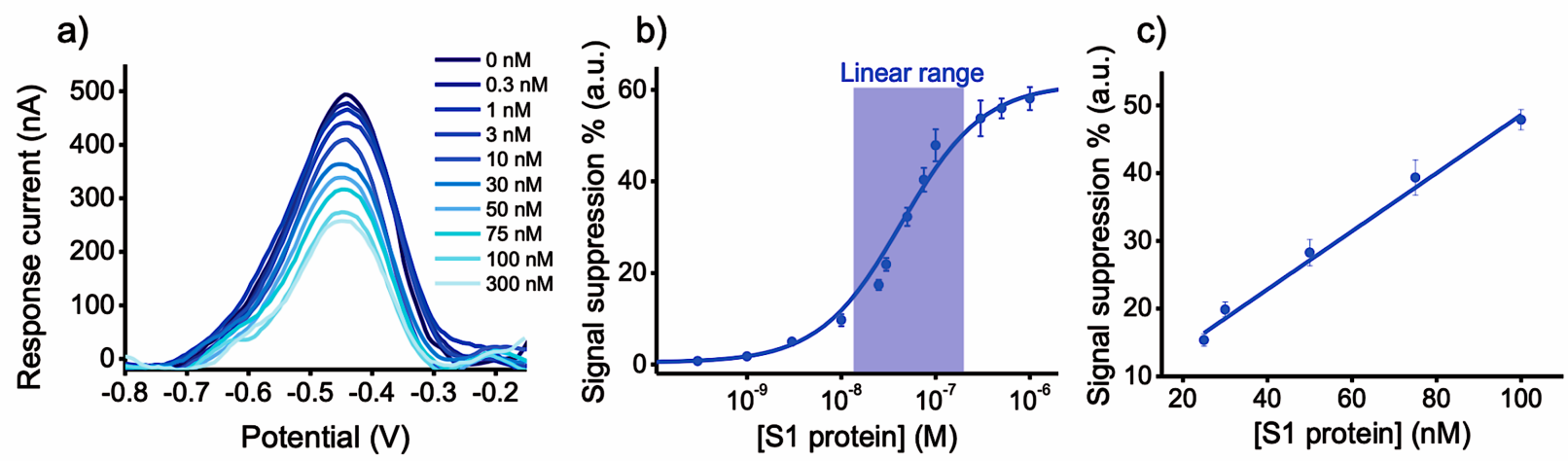

Figure 2. a) DPV voltammograms obtained in the presence of S1 SARS-CoV-2 protein in the concentration range 0.3 - $300 \mathrm{nM}$. b) Binding curve based on a Langmuir-type equation describing the response current as a function of the $S 1$ protein concentration. Highlighted is the concentration range in which response is linear. c) Calibration curve obtained by linear fit of the response current values in the $20-100$ nM S1 protein concentration range. (In all the figures data are reported as mean value $\pm S D, n=3$ ).

3.3. Specificity in the $\mathbf{S 1}$ protein-aptamer interaction. Further evidence of the interaction between the DNA aptamer and the target S1 protein, which supports the switching mechanism proposed for our aptasensor, was achieved by means of a competitive experiment in which the $\mathrm{S} 1$ protein $(100 \mathrm{nM})$ was pre-incubated in solution with a non-redox-tagged version of the same SARS-CoV-2 aptamer sequence, utilized as a competitor. Binding of this aptamer to the RBD of the target S1 protein would result in impeding subsequent binding to the redox-tagged aptamer on the electrode surface (Fig. 3a). When the S1 protein was pre-treated with the aptamer competitor, only a slight change in the current signal was observed compared with that in the absence of the protein (Fig 3b), and the signal suppression was only a fraction $(\sim 17 \%)$ of that obtained in the absence of the competitor at the same concentration of S1 protein (Fig. 3c). This suggests that the RBD of the $S 1$ protein was already occupied by the competitor inhibiting 
further binding, and the aptamer immobilized on the surface retained its conformational structure maintaining the redox reporter AttoMB2 close to the electrode surface.

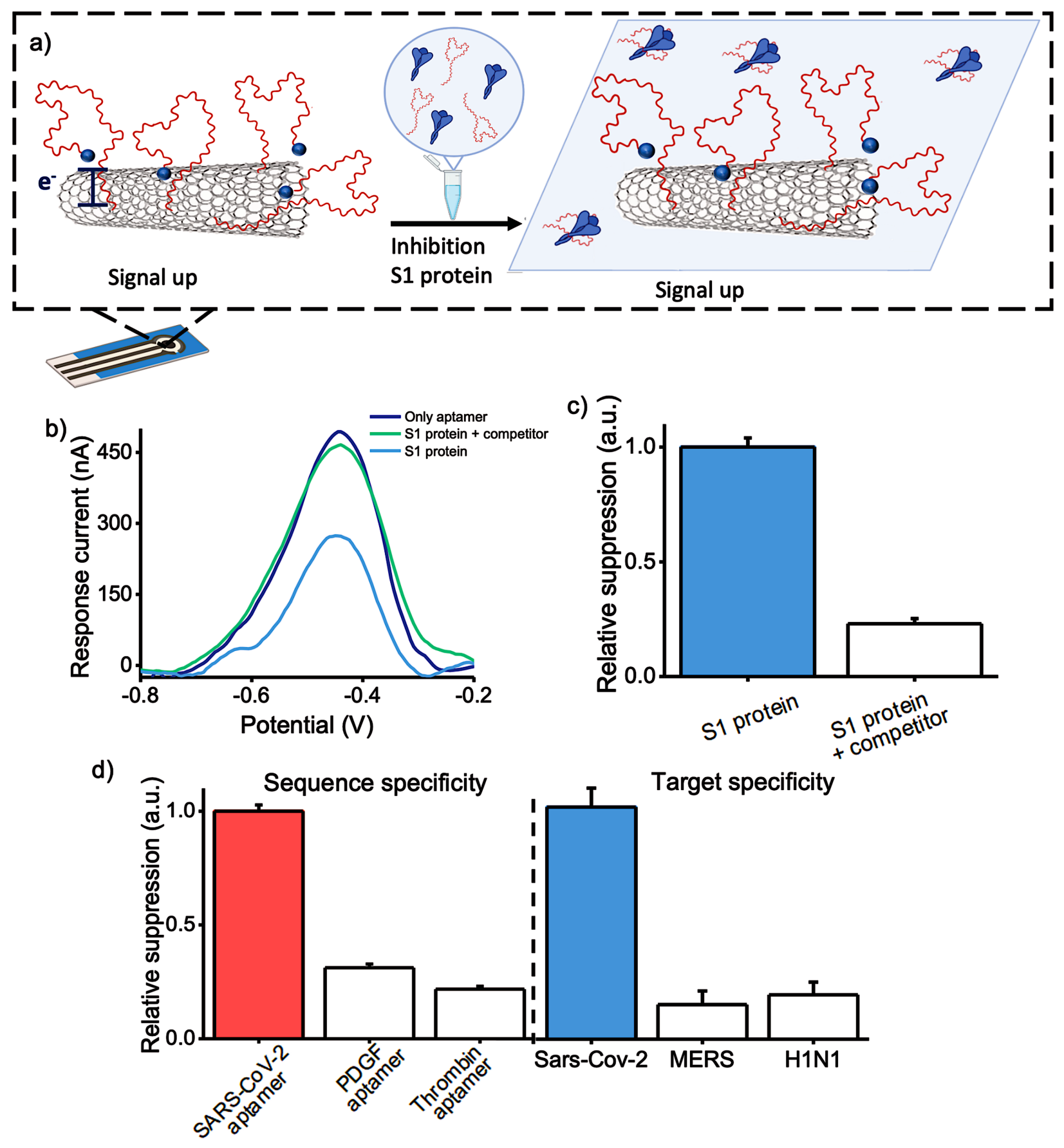

Figure 3. a) Schematic illustration of the aptasensor behavior when pre-treating the target $\mathrm{S} 1$ protein with the same SARS-CoV-2 aptamer, lacking the redox reporter, as a competitor in solution. b) DPV voltammograms in the absence of the target protein (only aptamer, dark blue 
line), in the presence of $100 \mathrm{nM} \mathrm{S1}$ protein pre-incubated with an excess of competitor aptamer (+ S1 protein + competitor, green line) and in the presence of the S1 protein at $100 \mathrm{nM}$ concentration (+ S1 protein, light blue line). c) Relative signal suppression obtained when measuring the amperometric current in the presence of S1 protein $100 \mathrm{nM}$ (light blue bar) and in the presence of the same protein incubated with the aptamer competitor (white bar). d) Specificity of the sensor evaluated by using different aptamer sequences immobilised onto the electrode surface (SARS-CoV-2, PDGF and thrombin aptamer) in the presence of S1 protein at $100 \mathrm{nM}$ concentration in PBS (left panel), and by using different proteins at $100 \mathrm{nM}$ concentration in PBS (S1 SARS-CoV-2, S1 MERS and H1N1) when the SARS-CoV-2 aptamer is immobilised onto the electrode surface (right panel). (In all the figures data are reported as mean value $\pm S D, n=3$ ).

We then tested the ability of our aptasensor to recognize its target $\mathrm{S} 1$ protein in a specific manner. To do so, we first exposed it to two different viral proteins from Middle East respiratory syndrome coronavirus (MERS-CoV) and Influenza A H1N1, respectively, as model potential interfering pathogens. At saturating concentrations $(100 \mathrm{nM})$, signal suppression of only $\sim 15 \%$ when using the MERS-CoV protein and of $\sim 19 \%$ when using the Influenza A H1N1 protein, respectively, were registered with respect to the signal suppression \% obtained with the target S1 protein (Fig. 3d right). To have further confirmation that the changes in the current signal were specific to binding of the S1 protein to its cognate aptamer ligand, we immobilized two different aptamer sequences, a thrombin DNA aptamer and a prostate-derived growth factor (PDGF) DNA aptamer, on the electrode surface, and exposed them to the S1 protein. We selected these aptamers because they had been previously used in the development of folding-based electrochemical sensors. ${ }^{41,42}$ In the presence of $100 \mathrm{nM}$ of S1 protein, a $15 \%$ signal suppression with the thrombin aptamer and a $21 \%$ signal suppression with the PDGF 
aptamer, with respect to the signal suppression obtained using the correct S1 protein aptamer couple, were observed, showing that cross-reactivity was minimal (Fig. 3d left).

3.4. S1 protein detection in VTM. To test the aptasensor ability to function in a more challenging matrix mimicking a real-world scenario, we investigated its performance when using S1 protein samples in viral transport medium (VTM), an artificial complex matrix used in the clinic for rapid antigen detection tests. Its composition includes physiologically balanced isotonic buffered solution at neutral $\mathrm{pH}$, a stabilizing protein component, and antibacterial and antifungal agents.

a)

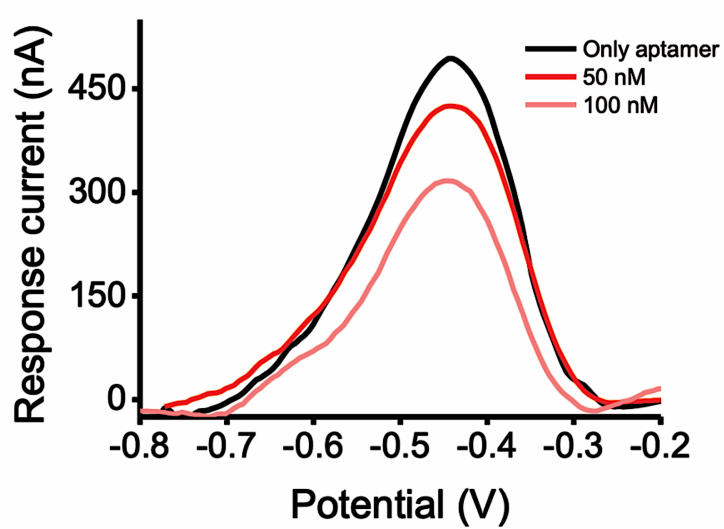

b)

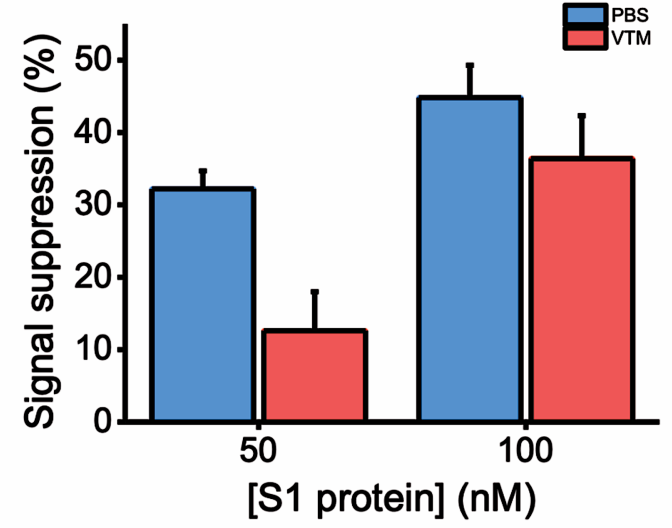

Figure 4. a) DPV voltammogram obtained in the presence of target $\mathrm{S} 1$ protein in VTM at 50 and $100 \mathrm{nM}$. b) Histograms showing the sensor performance, expressed as signal suppression \%, when the S1 protein is dissolved in PBS (blue bars) or VTM (red bars) (mean value $\pm S D, n=3$ ).

When the aptasensor was exposed to VTM-based solutions of S1 protein at concentrations of 50 and of $100 \mathrm{nM}$, a decrease in the measured current signal was observed (Fig. 2a). Values of signal suppression $\%$ of $15 \%$ and $36 \%$ were obtained, respectively (Fig. 2b). These values indicate that the aptasensor is still capable of 
recognizing and detecting the target $\mathrm{S} 1$ protein in a concentration-dependent manner, although the use of a complex matrix such as VTM has an impact on its analytical performances and leads to signal suppression values that are lower than those obtained using S1 protein samples in PBS (Fig. 4b), which is an effect described also for other COVID-19 biosensors. ${ }^{43}$

\section{CONCLUSIONS}

Folding-based E-DNAs that use target-induced changes in an aptamer structure have been previously designed that enabled protein detection on gold electrodes. ${ }^{27,44,45}$ Our work builds on this sensor format and proposes the use of cheap, highly conductive SWCNT-SPEs as a new substrate for aptamer-based E-DNAs. We have reported here the development of a rapid and reagent-free electrochemical sensing platform for the single-step detection of the SARS-CoV-2 S1 protein. We took advantage of the chemicalphysical properties of SWCNT-SPEs and engineered a folding-based mechanism that results in significant changes in the measurable amperometric current upon specific binding of the S1 protein to a DNA aptamer ligand. The obtained LOD and LOQ in the low nanomolar range, together with the high specificity for the target protein and the low cross-reactivity in the presence of interfering viral proteins, all suggest the potential employment of this aptasensor as a compact, easy-to-use sensing device for the detection of the $\mathrm{S} 1$ protein in a buffer solution or in a complex biological matrix, with different levels of sensitivity. The versatility and the simplicity of our design, in which a DNA aptamer as a specific, dynamic recognition element is combined with a CNT-based electrode substrate, could inspire the development of many more electrochemical 
platforms of this kind. As new aptamers designed to bind to desired target biomolecules can be produced by SELEX methods, a variety of new sensors can be envisioned with applications in a wide range of fields.

\section{AUTHOR INFORMATION}

\section{Corresponding Author}

* Alessandro Bertucci - Department of Chemistry, Life Sciences and Environmental Sustainability, University of Parma, 43124 Parma, Italy.

Email: alessandro.bertucci@unipr.it

\section{Author Contributions}

The manuscript was written through contributions of all authors. All authors have given approval to the final version of the manuscript.

\section{ACKNOWLEDGMENT}

This work was supported by the project "Biosensoristica innovativa per i test sierologici e molecolari e nuovi dispositivi PoCT per la diagnosi di infezione da SARS-CoV-2" funded in 2020 by "Bando Straordinario di Ateneo per Progetti di Ricerca Biomedica in ambito SARS-COV-2 e COVID-19" - University of Parma. This work has also benefited from the framework of the COMP-HUB Initiative, funded by the 'Departments of Excellence' program of the Italian Ministry for Education, University and Research (MIUR, 20182022). This research has financially been supported by the Programme "FIL-Quota Incentivante" of University of Parma and co-sponsored by Fondazione Cariparma (AB). 


\section{REFERENCES}

1. Hu, B.; Guo, H.; Zhou, P.; Shi, Z.-L. Characteristics of SARS-CoV-2 and COVID-19. Nat. Rev. Microbiol. 2021, 19 (3), 141-154.

2. World Health Organization. Coronavirus Disease (COVID-19) Situation Reports. https://www.who.int/emergencies/diseases/novel-coronavirus-2019/situation-reports

3. Kaye, A. D.; Okeagu, C. N.; Pham, A. D.; Silva, R. A.; Hurley, J. J.; Arron, B. L.; Sarfraz, N.; Lee, H. N.; Ghali, G. E.; Gamble, J. W.; et al. Economic Impact of COVID-19 Pandemic on Healthcare Facilities and Systems: International Perspectives. Best Pract. Res. Clin. Anaesthesiol. 2021, 35 (3), 293-306.

4. Leshem, E.; Wilder-Smith, A. COVID-19 Vaccine Impact in Israel and a Way out of the Pandemic. Lancet 2021, 397(10287), 1783-1785.

5. Thomas, S. J.; Moreira, E. D.; Kitchin, N.; Absalon, J.; Gurtman, A.; Lockhart, S.; Perez, J. L.; Pérez Marc, G.; Polack, F. P.; Zerbini, C.; et al. Safety and Efficacy of the BNT162b2 MRNA Covid-19 Vaccine through 6 Months. N. Engl. J. Med. 2021, 385 (19), 1761-1773. 6. Wilhelm, A.; Widera, M.; Grikscheit, K.; Toptan, T.; Schenk, B.; Pallas, C.; Metzler, M.; Kohmer, N.; Hoehl, S.; Helfritz, F. A.; et al. Reduced Neutralization of SARS-CoV-2 Omicron Variant by Vaccine Sera and Monoclonal Antibodies. medRxiv. 2021.

7. Chemaitelly, H.; Tang, P.; Hasan, M. R.; AlMukdad, S.; Yassine, H. M.; Benslimane, F. M.; Al Khatib, H. A.; Coyle, P.; Ayoub, H. H.; Al Kanaani, Z.; et al. Waning of BNT162b2 Vaccine Protection against SARS-CoV-2 Infection in Qatar. N. Engl. J. Med. 2021, 385 (24), e83. 
8. Lavezzo, E.; Franchin, E.; Ciavarella, C.; Cuomo-Dannenburg, G.; Barzon, L.; Del Vecchio, C.; Rossi, L.; Manganelli, R.; Loregian, A.; Navarin, N.; et al. Suppression of a SARS-CoV-2 Outbreak in the Italian Municipality of Vo'. Nature. 2020, 584 (7821), 425429.

9. Rosati, G.; Idili, A.; Parolo, C.; Fuentes-Chust, C.; Calucho, E.; Hu, L.; Castro E Silva, C. de C.; Rivas, L.; Nguyen, E. P.; Bergua, J. F.; et al. Nanodiagnostics to Face SARSCoV-2 and Future Pandemics: From an Idea to the Market and Beyond. ACS Nano. 2021. 10. Re-open EU https://reopen.europa.eu/en, and European Centre for Disease Prevention and Control, Diagnostic testing and screening for SARS-CoV-2 https://www.ecdc.europa.eu/en/covid-19/latest-evidence/diagnostic-testing

11. Eu Digital COVID Certificate. https://ec.europa.eu/info/live-work-traveleu/coronavirus-response/safe-covid-19-vaccines-europeans/eu-digital-covidcertificate_en

12. Morales-Narváez, E.; Dincer, C. The Impact of Biosensing in a Pandemic Outbreak: COVID-19. Biosens. Bioelectron. 2020, 163, 112274.

13. Zhu, H.; Zhang, H.; Ni, S.; Korabečná, M.; Yobas, L.; Neuzil, P. The Vision of Pointof-Care PCR Tests for the COVID-19 Pandemic and Beyond. Trends Analyt. Chem. 2020, 130, 115984.

14. Zhou, Y.; Wu, Y.; Ding, L.; Huang, X.; Xiong, Y. Point-of-Care COVID-19 Diagnostics Powered by Lateral Flow Assay. Trends Analyt. Chem. 2021, 145, 116452.

15. Peeling, R. W.; Olliaro, P. Rolling out COVID-19 Antigen Rapid Diagnostic Tests: The Time Is Now. Lancet Infect. Dis. 2021, 21 (8), 1052-1053. 
16. Mahshid, S. S.; Flynn, S. E.; Mahshid, S. The Potential Application of Electrochemical Biosensors in the COVID-19 Pandemic: A Perspective on the Rapid Diagnostics of SARS-CoV-2. Biosens. Bioelectron. 2021, 176, 112905.

17. Giannetto, M.; Bianchi, V.; Gentili, S.; Fortunati, S.; De Munari, I.; Careri, M. An Integrated loT-Wi-Fi Board for Remote Data Acquisition and Sharing from Innovative Immunosensors. Case of Study: Diagnosis of Celiac Disease. Sensors and Actuators B: Chemical. 2018, 273, 1395-1403.

18. Bianchi, V.; Mattarozzi, M.; Giannetto, M.; Boni, A.; De Munari, I.; Careri, M. A SelfCalibrating loT Portable Electrochemical Immunosensor for Serum Human Epididymis Protein 4 as a Tumor Biomarker for Ovarian Cancer. Sensors 2020, 20 (7), 2016.

19. Eissa, S.; Zourob, M. Development of a Low-Cost Cotton-Tipped Electrochemical Immunosensor for the Detection of SARS-CoV-2. Anal. Chem. 2021, 93 (3), 1826-1833. 20. Fabiani, L.; Saroglia, M.; Galatà, G.; De Santis, R.; Fillo, S.; Luca, V.; Faggioni, G.; D'Amore, N.; Regalbuto, E.; Salvatori, P.; et al. Magnetic Beads Combined with Carbon Black-Based Screen-Printed Electrodes for COVID-19: A Reliable and Miniaturized Electrochemical Immunosensor for SARS-CoV-2 Detection in Saliva. Biosens. Bioelectron. 2021, 171, 112686.

21. Li, J.; Lin, R.; Yang, Y.; Zhao, R.; Song, S.; Zhou, Y.; Shi, J.; Wang, L.; Song, H.; Hao, R. Multichannel Immunosensor Platform for the Rapid Detection of SARS-CoV-2 and Influenza A(H1N1) Virus. ACS Appl. Mater. Interfaces. 2021, 13(19), 22262-22270.

22. Li, D.; Song, S.; Fan, C. Target-Responsive Structural Switching for Nucleic AcidBased Sensors. Acc. Chem. Res. 2010, 43 (5), 631-641. 
23. Lin, M.; Song, P.; Zhou, G.; Zuo, X.; Aldalbahi, A.; Lou, X.; Shi, J.; Fan, C. Electrochemical Detection of Nucleic Acids, Proteins, Small Molecules and Cells Using a DNA-Nanostructure-Based Universal Biosensing Platform. Nat. Protoc. 2016, 11 (7), $1244-1263$.

24. Bonham, A. J.; Hsieh, K.; Ferguson, B. S.; Vallée-Bélisle, A.; Ricci, F.; Soh, H. T.; Plaxco, K. W. Quantification of Transcription Factor Binding in Cell Extracts Using an Electrochemical, Structure-Switching Biosensor. J. Am. Chem. Soc. 2012, 134 (7), 33463348.

25. Yousefi, H.; Mahmud, A.; Chang, D.; Das, J.; Gomis, S.; Chen, J. B.; Wang, H.; Been, T.; Yip, L.; Coomes, E.; et al. Detection of SARS-CoV-2 Viral Particles Using Direct, Reagent-Free Electrochemical Sensing. J. Am. Chem. Soc. 2021, 143 (4), 1722-1727.

26. Xiao, Y.; Lubin, A. A.; Heeger, A. J.; Plaxco, K. W. Label-Free Electronic Detection of Thrombin in Blood Serum by Using an Aptamer-Based Sensor. Angew. Chem. Int. Ed. 2005, 117 (34), 5592-5595.

27. Idili, A.; Parolo, C.; Alvarez-Diduk, R.; Merkoçi, A. Rapid and Efficient Detection of the SARS-CoV-2 Spike Protein Using an Electrochemical Aptamer-Based Sensor. ACS Sens. 2021, 6 (8), 3093-3101.

28. Sun, M.; Liu, S.; Wei, X.; Wan, S.; Huang, M.; Song, T.; Lu, Y.; Weng, X.; Lin, Z.; Chen, H.; Song, Y.; Yang, C. Aptamer Blocking Strategy Inhibits SARS-CoV-2 Virus Infection. Angew. Chem. Int. Ed. 2021, 60 (18), 10266-10272.

29. Zhang, Z.; Pandey, R.; Li, J.; Gu, J.; White, D.; Stacey, H. D.; Ang, J. C.; Steinberg, C.-J.; Capretta, A.; Filipe, C. D. M.; Mossman, K.; Balion, C.; Miller, M. S.; Salena, B. J.; Yamamura, D.; Soleymani, L.; Brennan, J. D.; Li, Y. High-Affinity Dimeric Aptamers 
Enable the Rapid Electrochemical Detection of Wild-Type and B.1.1.7 SARS-CoV-2 in Unprocessed Saliva. Angew. Chem. Int. Ed. 2021, 60 (45), 24266-24274.

30. Song, Y.; Song, J.; Wei, X.; Huang, M.; Sun, M.; Zhu, L.; Lin, B.; Shen, H.; Zhu, Z.; Yang, C. Discovery of Aptamers Targeting the Receptor-Binding Domain of the SARSCoV-2 Spike Glycoprotein. Anal. Chem. 2020, 92 (14), 9895-9900.

31. Li, J.; Zhang, Z.; Gu, J.; Stacey, H. D.; Ang, J. C.; Capretta, A.; Filipe, C. D. M.; Mossman, K. L.; Balion, C.; Salena, B. J.; Yamamura, D.; Soleymani, L.; Miller, M. S.; Brennan, J. D.; Li, Y. Diverse High-Affinity DNA Aptamers for Wild-Type and B.1.1.7 SARS-CoV-2 Spike Proteins from a Pre-Structured DNA Library. Nucleic Acids Res. 2021, 49 (13), 7267-7279.

32. Peinetti, A. S.; Lake, R. J.; Cong, W.; Cooper, L.; Wu, Y.; Ma, Y.; Pawel, G. T.; ToimilMolares, M. E.; Trautmann, C.; Rong, L.; et al. Direct Detection of Human Adenovirus or SARS-CoV-2 with Ability to Inform Infectivity Using DNA Aptamer-Nanopore Sensors. Sci. Adv. 2021, 7 (39), eabh2848.

33. Svobodova, M.; Skouridou, V.; Jauset-Rubio, M.; Viéitez, I.; Fernández-Villar, A.; Cabrera Alvargonzalez, J. J.; Poveda, E.; Bofill, C. B.; Sans, T.; Bashammakh, A.; Alyoubi, A. O.; O'Sullivan, C. K. Aptamer Sandwich Assay for the Detection of SARSCoV-2 Spike Protein Antigen. ACS Omega 2021, 6 (51), 35657-35666.

34. Chen, H.; Park, S.-K.; Joung, Y.; Kang, T.; Lee, M.-K.; Choo, J. SERS-Based DualMode DNA Aptasensors for Rapid Classification of SARS-CoV-2 and Influenza A/H1N1 Infection. Sensors Actuators B Chem. 2022, 355, 131324.

35. Kacherovsky, N.; Yang, L. F.; Dang, H. V; Cheng, E. L.; Cardle, I. I.; Walls, A. C.; McCallum, M.; Sellers, D. L.; DiMaio, F.; Salipante, S. J.; Corti, D.; Veesler, D.; Pun, S. 
H. Discovery and Characterization of Spike N-Terminal Domain-Binding Aptamers for Rapid SARS-CoV-2 Detection. Angew. Chem. Int. Ed. 2021, 60 (39), 21211-21215.

36. Rasheed, P. A.; Sandhyarani, N. Carbon Nanostructures as Immobilization Platform for DNA: A Review on Current Progress in Electrochemical DNA Sensors. Biosens. Bioelectron. 2017, 97, 226-237.

37. Wang, J. Carbon-Nanotube Based Electrochemical Biosensors: A Review. Electroanalysis 2005, 17 (1), 7-14.

38. Fortunati, S.; Rozzi, A.; Curti, F.; Giannetto, M.; Corradini, R.; Careri, M. Novel Amperometric Genosensor Based on Peptide Nucleic Acid (PNA) Probes Immobilized on Carbon Nanotubes-Screen Printed Electrodes for the Determination of Trace Levels of Non-Amplified DNA in Genetically Modified (GM) Soy. Biosens. Bioelectron. 2019, 129, 7-14.

39. Arroyo-Currás, N.; Scida, K.; Ploense, K. L.; Kippin, T. E.; Plaxco, K. W. High Surface Area Electrodes Generated via Electrochemical Roughening Improve the Signaling of Electrochemical Aptamer-Based Biosensors. Anal. Chem. 2017, 89 (22), 12185-12191. 40. Keighley, S. D.; Li, P.; Estrela, P.; Migliorato, P. Optimization of DNA Immobilization on Gold Electrodes for Label-Free Detection by Electrochemical Impedance Spectroscopy. Biosens. Bioelectron. 2008, 23 (8), 1291-1297.

41. Lai, R. Y.; Plaxco, K. W.; Heeger, A. J. Aptamer-Based Electrochemical Detection of Picomolar Platelet-Derived Growth Factor Directly in Blood Serum. Anal. Chem. 2007, 79 (1), 229-233. 
42. Centi, S.; Tombelli, S.; Minunni, M.; Mascini, M. Aptamer-Based Detection of Plasma Proteins by an Electrochemical Assay Coupled to Magnetic Beads. Anal. Chem. 2007, $79(4), 1466-1473$.

43. Seo, G.; Lee, G.; Kim, M. J.; Baek, S.-H.; Choi, M.; Ku, K. B.; Lee, C.-S.; Jun, S.; Park, D.; Kim, H. G.; Kim, S.-J.; Lee, J.-O.; Kim, B. T.; Park, E. C.; Kim, S. II. Correction to Rapid Detection of COVID-19 Causative Virus (SARS-CoV-2) in Human Nasopharyngeal Swab Specimens Using Field-Effect Transistor-Based Biosensor. ACS Nano 2020, 14, 4, 5135-5142.

44. Lubin, A. A.; Plaxco, K. W. Folding-Based Electrochemical Biosensors: The Case for Responsive Nucleic Acid Architectures. Acc. Chem. Res. 2010, 43 (4), 496-505.

45. Leung, K. K.; Downs, A. M.; Ortega, G.; Kurnik, M.; Plaxco, K. W. Elucidating the Mechanisms Underlying the Signal Drift of Electrochemical Aptamer-Based Sensors in Whole Blood. ACS Sensors 2021, 6 (9), 3340-3347. 